\title{
Frontiers of (Pareto) Optimal and Sustainable Water Management for Hydropower and Ecology
}

\begin{abstract}
Paolo Perona ${ }^{1,2,4 *}$, Amin Niayifar $^{3}$, Robin Schwemmle ${ }^{2,5}$, Pierre Razurel ${ }^{2}$, Reto Flury ${ }^{4}$, Elias Winz ${ }^{4}$ and D. A. Barry ${ }^{1}$

${ }^{1}$ Ecological Engineering Laboratory (ECOL), Institute of Environmental Engineering (IIE), School of Architecture, Civil and Environmental Engineering (ENAC), EPFL, Lausanne, Switzerland, ${ }^{2}$ School of Engineering, Institute for Infrastructure and Environment, The University of Edinburgh, Edinburgh, United Kingdom, ${ }^{3}$ Stream Biofilm and Ecosystem Research Laboratory (SBER), Institute of Environmental Engineering (IIE), School of Architecture, Civil and Environmental Engineering (ENAC), EPFL, Lausanne, Switzerland, ${ }^{4} \mathrm{HOL}$ INGER AG, Urban Drainage and Hydraulic Engineering, Bern, Switzerland, ${ }^{5}$ Chair of Hydrology, University of Freiburg, Freiburg, Germany
\end{abstract}

River regulation alters the natural flow regime of streams with consequent impacts on terrestrial and aquatic habitats of the riparian zone. The severity of such impacts can be modulated by changing the redistribution rules at water intakes and reservoirs. Contrary to minimal-flow policies, non-proportional and proportional redistribution policies result in variable environmental flow releases, namely Dynamic Environmental Flows (DEFs), which improve the global (i.e., ecological and economic) efficiency of water use practice, e.g., for energy production. DEF assessment is based on different indicators. However, the choice and aggregation method of different hydrological and fish habitat indicators affects the assessment of the global power plant performance, i.e., the Frontier of efficient solutions (sensu Pareto). This study investigates DEF assessment, and shows the extent to which the choice and method of aggregation of different indicators impacts the Frontier of Paretoefficient solutions. The findings are supported by six case studies of hydropower practice that differ in terms of river morphology, energy production amount and technique. The relative importance of several types of indicators is examined as is their influence on optimal and sustainable water allocation solutions that lie on the Pareto Frontier. The analysis shows that DEFs arising from either proportional or non-proportional redistribution rules can positively impact strategies of sustainable management of freshwater resources.

Keywords: dynamic environmental flows, hydropower, sustainable water management, pareto frontier, water use, non-proportional redistribution, fermi functions

\section{INTRODUCTION}

Energy from renewable sources has gradually grown over recent decades in order to promote the transition towards green forms of production and so mitigate global warming. Among these, hydropower is an attractive alternative to fossil fuel technologies (Hertwich et al., 2015), notwithstanding that associated carbon dioxide emissions and environmental impact are subject to debate (Abbasi and Abbasi, 2011; Abbasi et al., 2011; Almeida et al., 2019). Regardless of this, further development of hydropower

Abbreviations: DEF, Dynamic Environmental Flows; FOEN, (Swiss) Federal Office of the ENvironment; HP, Hydropower Plant; IHA, Indicators of Hydrological Alteration; MRF, Minimal Flow Release; PHABSIM, Physical HABitat SIMulation model; PP, Proportional Policies; NPP, Non-Proportional Policies; SEPA, Scottish Environment Protection Agency; SHP, Small Hydropower Plant; WUA, Weighted Usable Area. 
will increase the exploitation of surface waters and so too the anthropic pressure on freshwater ecosystems. Therefore, the development of ecologically sustainable water management policies remains a major challenge for management of hydropower schemes (Manzano-Agugliaro et al., 2017).

The impact of hydropower on river ecosystems depends largely on the water redistribution rule adopted to release environmental flows (Robson et al., 2011; Benejam et al., 2016; Gabbud and Lane, 2016). Typically, environmental flows correspond to a static redistribution rule determining a constant flow release (Minimal Flow Requirement, MFR, or Residual Flow), which is determined from the flow duration curve of the natural flow regime. The constant flow release MFR is known to adversely affect both the morphological and ecological status of riverine ecosystems, mainly due to its incompatibility with the variability of the natural flow regime (Arthington et al., 2006; Poff et al., 2007; Petts, 2009; Meijer et al., 2012). Indeed, the premise is that all flow quantiles of the natural flow regime (high, medium and low discharges) are important, unlike the static rules that are typical of MRF policies (Moyle and Mount, 2007). Some countries currently apply proportional policies (PP) as redistribution rules, which better mimic stream natural variability. For example, these rules are included in the environmental law of Scotland and England (Environment Agency, 2013; SEPA, 2014; Willmott et al., 2016) and represent a substantial improvement compared to the static MFR rules that are still used in many European countries (Basso and Botter, 2012; Gorla and Perona, 2013; Lazzaro et al., 2013; Perona et al., 2013; Razurel et al., 2018).

Non-Proportional flow release Policies (NPP) (Razurel et al., 2016, 2018; Niayifar and Perona, 2017) are a new class of dynamic distribution rules that generate environmental flows with variability (hydrological characteristics such timing and magnitude, for instance) that mimics the unaltered system. Compared to PPs, the fraction of water released to the riverine environment with NPPs varies with the incoming flow rate, guaranteeing more flexibility and opportunities to improve both the ecologic status and hydropower productivity as shown by the so-called efficiency plot (Razurel et al., 2018). In this plot, efficient solutions for which no further improvement of both quantities is possible form a borderline, which is known as the "Pareto Frontier". The general mathematical representation of the NPP function is based on four parameters that determine the form of the redistribution function [see Stream-scale model and allocation scenarios and (Razurel et al., 2016) for details]. This leads to considerable flexibility in the latter, and allows for systematic exploration of a wide range of water allocation policies. The performance of such policies is quantified through environmental indicators and the amount of energy produced plotted in pair in the efficiency plot. The use of an optimal NPP strategy in preference to MFR or PP approaches results in the same energy production with reduced anthropic pressure on the river system (Razurel et al., 2018).

The (Pareto) Frontier of optimal redistribution rules depends on the definition of the environmental indicator. This indicator is usually built by aggregating together many equally-weighted indicators of different categories, grouped as hydrological and fish suitability categories (Mathews and Richter, 2007; Poff et al.,
2010). Thus, it is usually not possible to identify the effect of a single contribution to the global indicator. Here, the consequences of applying variable (rather than fixed) weightings are explored by: 1) investigating the impact of fish habitat suitability indicators on the efficiency plot in comparison to hydrological indicators; 2) analyzing the role of each IHA indicator in order to identify those with the highest importance; 3) proposing two alternative procedures for assessing fish habitat suitability and quantifying their role in defining the Pareto Frontier; 4) quantifying the role of uncertainties on the discharge time sequence as well as on the Pareto Frontier. The latter point is explored using a nonlinear hydrological model that overcomes the lack of data that is often found in practice. The efficiency plot is numerically built using data from five Small Hydropower Plants (SHPs) (without storage capacity) and one traditional Hydropower Plant (HP) (with storage capacity) from three different countries: Switzerland, United Kingdom and Italy.

\section{METHODOLOGY}

\subsection{Stream-Scale Model and Allocation Scenarios}

Consider Figure 1A, where a diversion node provides water to a powerhouse for energy production. The allocation scenario represents the policy used to split the natural flow rate $I$ upstream to the water intake rates between the hydropower plant $Q_{H P}$ and the river reach $Q_{e n v}$, i.e.,

$$
I=Q_{H P}+Q_{e n v} \text {. }
$$

The flow rate $Q_{e n v}$ is defined as:

$$
Q_{e n v}=\left(I-Q_{\min }\right) f_{e n v}+Q_{m f r},
$$

with $f_{\text {env }}$ being the non-proportional coefficient determining the fraction of water that is left to the river; $Q_{\min }$ is the minimum workable flow; and $Q_{m f r}$ is the minimal flow release, which is guaranteed for any flow allocated to the hydropower turbine. The case of traditional hydropower fed by water stored into a reservoir was analyzed by Niayifar and Perona (2017). The main difference with the present theory is that in their case Eq. $\mathbf{1}$ is a differential equation for the stored volume so that the whole optimization problem requires machine-learning techniques, e.g., multiobjective evolutionary algorithms.

Here, different policies are simulated, the first one being the Minimal Flow Release $\left(Q_{m f r}\right)$. This flow release is prescribed by law in many countries. In Switzerland, for example, legislation prescribes the use of the discharge observed in the river at least 347 days per year, referred to as $Q_{347}$. The MFR is a static policy that tends to maximize energy production in spite of riparian ecological needs. It will be used as the reference scenario for comparison with other policies. The second group of allocation scenarios involves proportional distributions, which is a policy used in the United Kingdom. In this case, the flow rate diverted to the environment is a fixed percentage of the incoming flow, which is added to the $Q_{m f r}$. In other words, $f_{\text {env }}$ in Eq. 2 is a constant taking a value between 0 and 1 . Notice that the MFR policy 

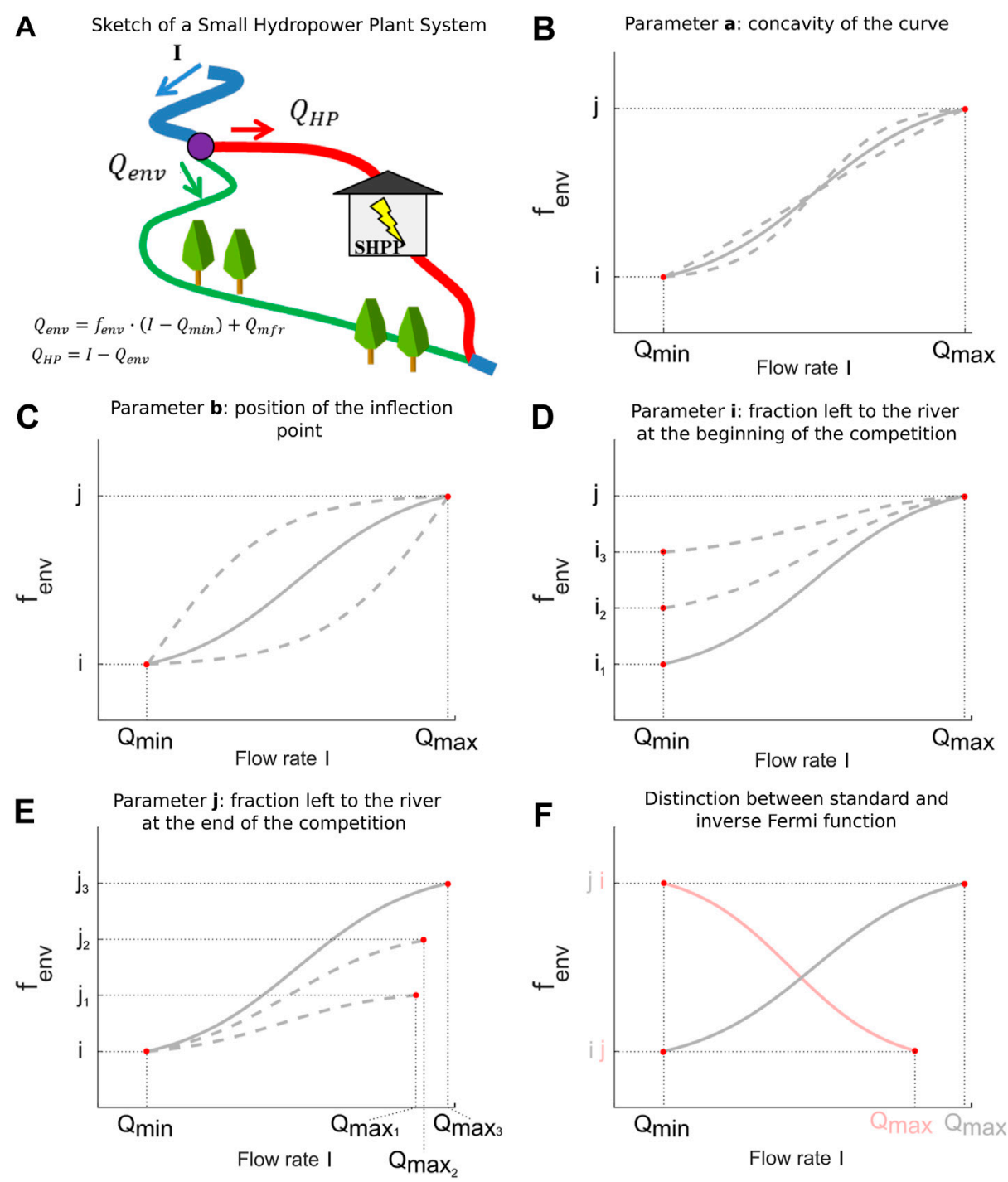

FIGURE 1 | (A) Sketch of a run-of-the-river SHP. A part of the natural incoming flow at the intake I (blue line)is diverted to the powerhouse $Q_{h p}$ (red line). The remaining flow rate $Q_{e n v}$ is left to the river (green line). (B)(C)(D)(E) Influence of the parameters $a, b, i$ and $j$ on the shape Fermi function. (F) Distinction between the standard Fermi function (gray curve) and the inverse Fermi function (pink curve).

corresponds to a particular case of proportional policies for which $f_{e n v}=0$. The last class of allocation scenarios consists of nonproportional policies, which were proposed to improve the ecological efficiency of proportional redistribution (Razurel et al., 2016). Unlike the previously presented redistribution rules, for non-proportional distribution the fraction of water released to the river, $f_{\text {env }}$, depends on the value of the incoming flow rate $I$, and is modeled as a class of nonproportional Fermi functions (Lifshitz and Landau, 1984) as follows (Razurel et al., 2016, 2018):

$$
f_{\text {env }}(I)=\left[1-M-\frac{Y}{\exp [a(I-b)]+1}\right](j-i)+i,
$$

with $\quad M=A /(A-1), \quad Y=(1-M)[\exp (-a b)+1] \quad$ and $A=[\exp (-a b)+1] /[\exp [a(1-b)]+1]$. This equation represents the competition for water between the environment and the hydropower plant occurring within the range $\left[Q_{\min }, Q_{\max }\right]$ (Perona et al., 2013), which depends on the characteristics of the turbine, viz., the minimum workable flow $Q_{H P, \min }$, and the design flow $Q_{H P, \max }=Q_{N}$. The non-proportional distribution is based on the choice of the four parameters $a, b, i, j$, which determine the shape of the redistribution function and thus how much water is allocated (Figures 1B-F). The parameter $a$ controls the concavity of the curve, $b$ controls the position of the inflection point, $i$ is the fraction of water released to the river at the beginning of the allocation period at $Q_{\text {min }}=Q_{m f r}+Q_{H P, \min }$ and $j$ is the fraction of water released to the river at the end of the allocation period at $Q_{\max }$. Two different types of functions can be distinguished. When $i<j, f_{\text {env }}$ is called a standard distribution function, shown in gray in Figure 1F) and otherwise $i>j$ and $f_{e n v}$ represents an inverse function, shown in pink in Figure 1F). 
TABLE 1 | Summary of hydrological parameters used in the Indicators of Hydrologic Alteration and their characteristics.

\section{IHA statistics group}

Group 1: Magnitudes of Monthly water conditions

Group 2: Magnitudes and Duration of annual extreme Water conditions

\section{Regime Characteristics}

Magnitude Timing

Magnitude Duration
Group 3: Timing of annual Extreme water conditions

Group 4: Frequency and Duration of high and low Pulses

Group 5: Rate and frequency Of water condition changes
Timing

Magnitude Frequency Duration

Frequency Rate of change
Hydrologic parameters

Mean value for each calendar month

Annual minimum 1-d meano

Annual maximum 1-d meano

Annual minimum 3-days meano

Annual maximum 3-days meano

Annual minimum 7-days meano

Annual maximum 7-days meano

Annual minimum 30-days meana

Annual maximum 30-days meano

Annual minimum 90-days meano

Annual maximum 90-days mean Julian date of annual 1-d maximum Julian date of annual 1-d minimum

No. of high pulses each year No. of low pulses each year Mean duration of high pulses per year Mean duration of low pulses per year Means of all positive differences Between consecutive daily means Means of all negative differences Between consecutive daily values No. of rises

No. of falls

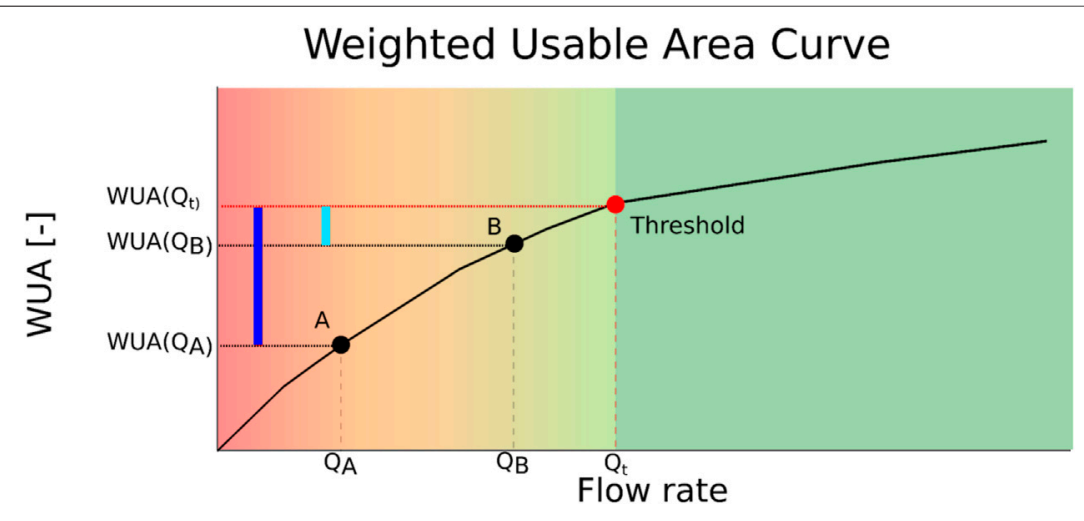

FIGURE 2 | Example of a Weighted Usable Area curve modeled with the PHABSIM software (Bloesch et al., 2005; Milhous et al., 1984; Maddock, 1999). The wellbeing of the fish is shown by the color of the background, green being good and red is bad. It is used to compute the three alternatives of fish habitat suitability indicators $\mathrm{ECO}_{\mathrm{V} 1}, \mathrm{ECO}_{\mathrm{V} 2}$ and $\mathrm{ECO}_{\mathrm{V}}$.

In order to compare static (i.e., MFR based) and dynamic (e.g., proportional and non-proportional) policies, and to evaluate their performance, a synthetic dimensionless indicator is employed (Razurel et al., 2016, 2018). This ecohydrological indicator is obtained by aggregating the 32 Indicators of Hydrological Alteration (IHA) of Richter et al. (1996), which allows a composite assessment of flow variability (Table 1) and fish habitat suitability. Fish habitat suitability is typically represented by Weighted Usable Area (WUA) curves as modeled with the PHABSIM software (Milhous et al., 1984; Maddock, 1999; Bloesch et al., 2005). Whilst we refer to Razurel et al. (2016) and Razurel et al. (2018) for details, here it suffices to say that Weighted Usable Area (WUA) curves were computed for one dominant fish species, the brown trout, discriminating between juveniles and adults. Although this fish species is not the predominant one in all examined case studies, it better represents common features of the species involved. In particular, we used WUA curves in order to identify the threshold flow rate $Q_{t}$ (red point in Figure 2) around which criteria for penalizing flow conditions where set (see Sensitivity Analysis).

The Indicators of Hydrologic Alteration (IHA) method (Richter et al., 1996), which is based on analysis of hydrological data, defines a set of indicators used to quantify the degree of alteration caused in by the diversion of the water sent to the hydropower plant. As shown in the Table 1, the 32 IHA indicators are sorted into five groups characterizing annual 
TABLE 2 | Parameters of the six case studies compared for the sensitivity analysis.

\begin{tabular}{|c|c|c|c|c|c|c|}
\hline Location & Turbine & $\mathbf{Q}_{\mathbf{H P}, \min }$ & $\mathbf{Q}_{\mathbf{N}}$ & $\mathbf{Q}_{\mathrm{mfr}}$ & Power & Energy Production \\
\hline- & Type & - & - & - & - & - \\
\hline- & - & {$\left[\mathrm{m}^{3} / \mathrm{s}\right]$} & {$\left[\mathrm{m}^{3} / \mathrm{s}\right]$} & {$\left[\mathrm{m}^{3} / \mathrm{s}\right]$} & {$[k W]$} & [GWh] \\
\hline Buseno (SHP) & Cross-flow & 0.45 & 4.5 & 0.38 & 2,340 & 8.8 \\
\hline Cauco (SHP) & Cross-flow & 0.35 & 3.5 & 0.315 & 1,390 & 5.0 \\
\hline Ponte Brolla (SHP) & $2 \times$ Francis & 1.2 & 12 & 0.55 & 1900 & 13.9 \\
\hline Puni (SHP) & Cross-flow & 0.017 & 1 & 0.125 & 3,740 & 10 \\
\hline Inverliever (SHP) & Francis & 0.02 & 0.95 & 0.07 & 550 & 2 \\
\hline Traditional HP with storage & Cross-flow & - & 2 & 0.18 & - & 2.3 \\
\hline
\end{tabular}

hydrological variations such as magnitude, timing, duration, frequency and rate of change of discharges. In the original stream-scale model (Razurel et al., 2018), all the indicators were given the same weight to compute the final indicator. In this work, we evaluate the influence of each of them on the efficiency plot, i.e., they are all treated separately with no final aggregation.

The economic indicator is defined as the energy production normalized to the maximal production obtained with the $Q_{m f r}$ policy. This indicator is therefore presented as a dimensionless number ranging between 0 and 1 . We deliberately decided not to present it in terms of energy price in order to avoid the subjective effects played by market fluctuations, which would also it make difficult to compare power plant operations from different countries.

After the environmental and economic indicators are calculated for each water allocation policy scenario, the efficiency plot is built by plotting the energy production $v s$ the environmental indicator. Optimal water allocation policies are then identified as the uppermost points, whose sequence in the efficiency plot forms a curve called the Pareto Frontier. By definition, the Pareto Frontier represents the limit where it is not possible to improve a scenario by making an indicator better without making another one worse (Marler and Arora, 2004). In the efficiency plot, we chose to represent only the Pareto Frontier, in addition to the MFR policy, and not the results for the suboptimal water allocation scenarios in order to make the comparison between the different hydrological sub-indicators clear. The sub-optimal policies all lie below the Frontier (Razurel et al., 2016, 2018).

\subsection{Case Studies}

Five run-of-the-river SHP and one traditional hydropower plant (i.e., with storage) subjected to different hydrological regimes, were investigated. Technical details including the turbine nominal flow, $Q_{N}$ are provided in Table 2. Buseno, Cauco and Ponte Brolla (Razurel et al., 2018) are located in Southern Switzerland, Puni in South Tyrol in Italy and Inverliever in the West Coast of Scotland. For Buseno, Cauco, Ponte Brolla and Puni, we used 29-y hydrographs measured by the Swiss Federal Office for the Environment (FOEN), which were transposed to each site following Gorla and Perona (2013) and Razurel et al. (2018). For Inverliever, the natural streamflow series was reconstructed based on historical data of flow rate allocated to the SHP and overflow data at the water intake. The traditional
TABLE 3 | Definition of the three alternatives compared in the sensitivity analysis to evaluate the fish habitat suitability indicator ( $d$ corresponds to the number of consecutive days under the threshold).

\begin{tabular}{ll} 
Alternative & \multicolumn{1}{c}{ Definition } \\
\hline EcoV1 & $\max \left(\sum\right.$ consecutive days under threshold $)$ \\
EcoV2 & $\max \left(\sum_{i=1}^{d}\left(W \cup A\left(Q_{i}\right)-W U A\left(Q_{t}\right)\right)^{2}\right)$ \\
EcoV3 & $E_{\text {co } 1} \sum$ (total days under threshold $) /($ total number of days $)$
\end{tabular}

hydropower plant considered here is a synthetic, demonstrative case based on the re-scaled natural flow regime of the Maggia river in southeast Switzerland (Niayifar and Perona, 2017).

\subsection{Sensitivity Analysis}

The first goal of the sensitivity analysis is to identify, for each case study, which IHAs produce the efficiency plot with the highest variability, i.e., the IHAs that are the most affected by the distribution policies. To do so, the efficiency plot was computed considering the effect of each hydrological subindicator separately. Then, the Pareto frontiers of each of them are compared.

The other parameter used for the computation of the ecohydrological indicator concerns fish habitat suitability. This is used to assess the suitability of the environmental flow regime for selected fish species, i.e., via the WUA curve (Capra et al., 1995). For increasing streamflow magnitude, a break-point appears in the slope of the WUA curve, which is generally assumed as the threshold minimal flow below which habitat conditions for that fish species are critical. In previous studies, this fish ecological indicator, $E c O_{V 1}$, was expressed as the maximum number of consecutive days under the threshold over the whole time series (Razurel et al., 2016), which corresponds to the longest stress period for the fish (Payne, 2003) (Figure 2). In this work, two alternatives are proposed in order to make the fish habitat suitability indicator more representative. The first alternative, $E \mathrm{CO}_{V 2}$, introduces a penalty as a function of distance from the threshold, such that staying far below the threshold for a short time can be more harmful for the fish than staying a longer time just below the threshold. The second alternative, $\mathrm{Eco}_{V 3}$, includes a term corresponding to the total fraction of days under threshold for the entire time series. This differentiates scenarios where falling only once under the threshold for the whole time series results into a worse indicator in comparison to scenarios with a hydrograph staying very close to the threshold for a significant amount of time (alternately 

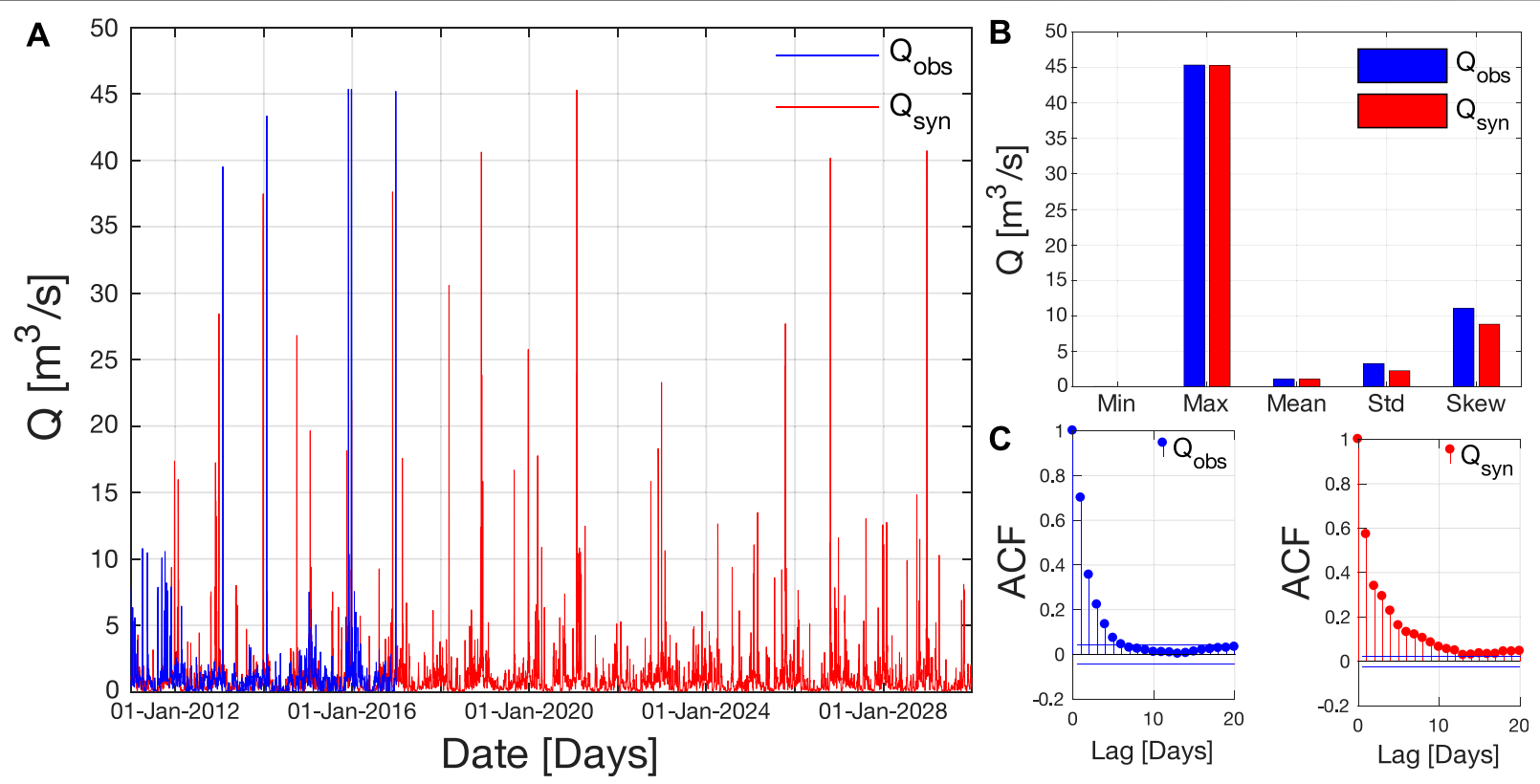

FIGURE 3 | (A) Example of synthetic 20-y hydrograph obtained with the back-filtered PAR model based on the 5-y hydrograph for the Inverliever SHP. (B) Comparison of some statistical quantities for the observed and synthetic hydrographs. (C) Comparison of the autocorrelation function of the observed and synthetic hydrographs.

above and below). The three alternatives $\mathrm{Eco}_{V 1}, \mathrm{Eco}_{V 2}$ and $\mathrm{Eco}_{V 3}$ are detailed in Table 3.

\subsection{Synthetic Data Generation}

A common problem arising in the analysis of real case studies is the lack of historical data. For the case of Inverliever, for example, only $6 \mathrm{y}$ of hydrological data are available, whereas the IHA method would recommend a minimum of $20 \mathrm{y}$. To overcome this problem, a 20-y synthetic hydrograph was generated based on the available data series. For this, a back-filtered Periodic Autoregressive Model (PAR) was used (Brockwell et al., 2002). Preliminary transformations to the original series were performed: logarithmic transformation followed by removal of trends and shifts by daily standardization. The model order was then determined using the temporal partial autocorrelation function. After this, the synthetic time series was generated with the AR model, the trend was added back and the series re-transformed. As the synthetically generated time series was still particularly noisy at low-medium flow rates, a moving average filter was applied. In particular, the size of the moving average window was determined with a threshold conserving the water balance and by minimizing the differences for the temporal autocorrelation function and other statistical quantities (minimum flow, maximum flow, mean flow, standard deviation, skewness coefficient) between the observed and generated time series. To validate the model and to compare the generated synthetic hydrograph with the historical data, we also computed the IHAs as non-linear evaluation parameters for both time series. An example of generated synthetic hydrograph is shown in Figure 3.

\section{RESULTS}

\subsection{Sensitivity Analysis of the Indicators of Hydrological Alteration}

Figure 4 shows the Pareto frontiers obtained when considering each hydrological sub-indicator separately. Colors are used to make the distinction between the different groups. There are five groups in total (Table 1); however, as far as the SHPs are concerned, the third group is not considered in the evaluation because its inclusion would bias the assessment in favor of the release policy. In fact, given the absence of storage, the Julian date of annual 1-d maximum and minimum will be the same for the pre- and post-impact series, so that all policies would have the same impact. For the SHPs we see that most of the Pareto frontiers are almost horizontal and close to unity. This suggests that the IHAs used to assess the location of the Frontier are not affected by the change in water allocation policy. Their values are close to those corresponding to the natural flow regime policy, whence their ineffectiveness for the Frontier. On the other hand, some other IHAs markedly affect the form of the Frontier given that their value is strongly affected by the chosen water redistribution policy.

IHA 16 affects four out of five SHP case studies (Figures 4A-E). In the original classification of Richter et al. (1996), this indicator corresponds to the highest 3-days value flow rate occurring during the year and is a measure of environmental stress disturbance.

For the five SHP case studies, the Pareto frontiers associated with IHAs of Groups 1 and 2 show more variability than those for Groups 4 and 5. Group 1 includes 12 parameters, each of which 

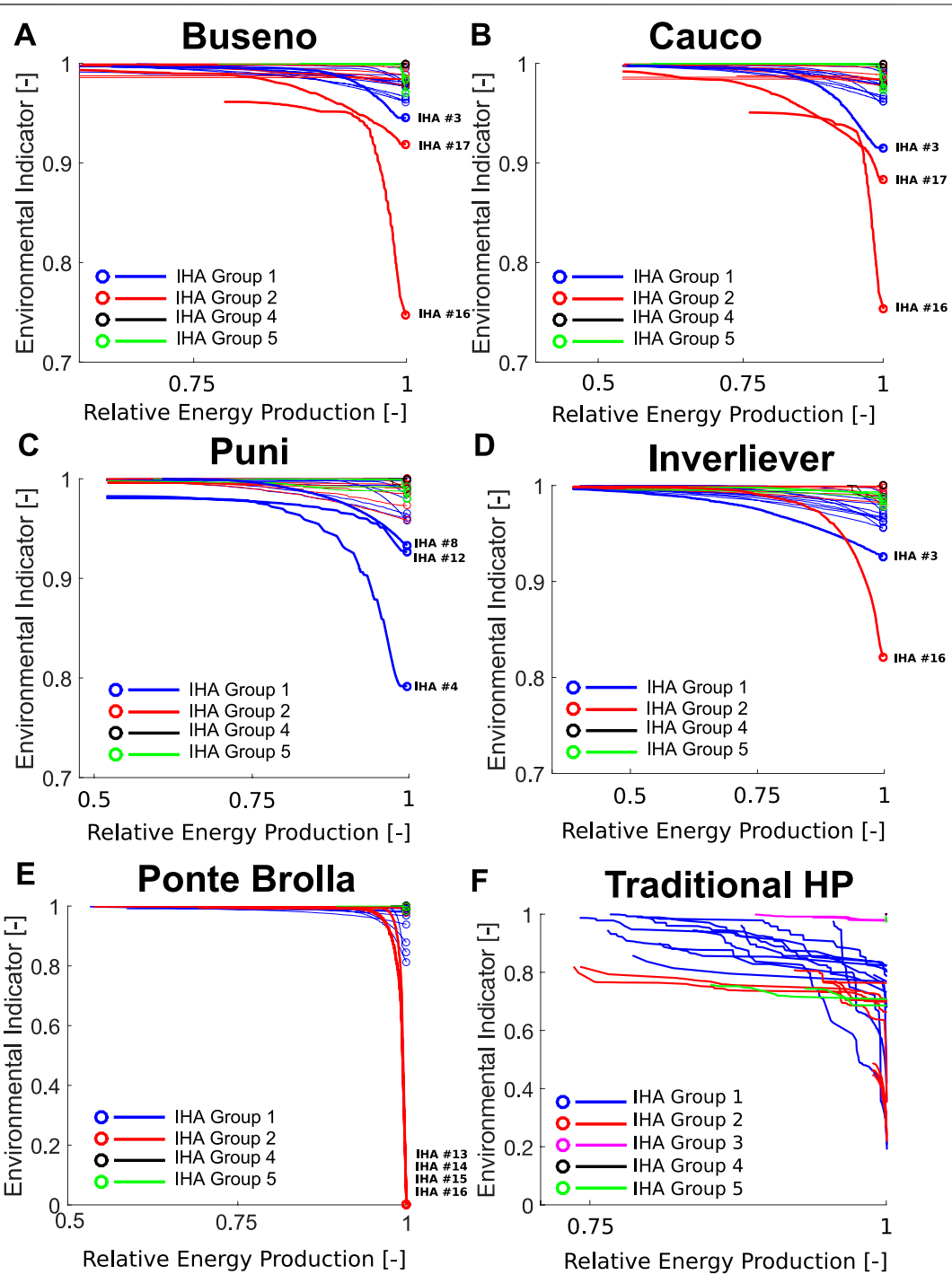

FIGURE 4 | Pareto Frontier resulting from non-proportional policies for each of the $32 \mathrm{IHA}$ sub-indicators. Dots represent $Q_{m f r}$ policies. Each color refers to one of the five groups of hydrological sub-indicators described in Table 1. Plots (A),(B),(C),(D) and (E) show the results for five different SHP and (F) for a traditional Hydropower Plant. The widest curves represent the IHA with the highest variability for each case study, as summarized in Table 4.

measures the mean of the daily water conditions for a given month. It thus provides a general measure of habitat availability or suitability. Group 2 corresponds to the magnitudes and duration of annual extreme water conditions (ranging from daily to seasonal). It also provides measures of environmental stress and disturbance during the year. According to Richter et al. (1996), the Group 2 indicators reflect stress conditions affecting the reproduction of certain fish species.

For the case of the traditional hydropower plant with storage, except for Group 3 indicators, all sub-indicators clearly influence the Pareto Frontier. This means that traditional hydropower plants have a greater ecological impact than SHPs for changing hydrologic regimes, as expected. Hence, while "diverting water" alone is mainly detected by Groups 1 and 2, adding water storage also impacts indicators of Groups 4 and 5 .
The next step of the sensitivity analysis is to consider only a few hydrological sub-indicators to re-calculate the Pareto Frontier. That is, only IHAs with the highest impact are considered when computing the environmental indicator. Results are shown in Figure 5 for the power plants of Inverliever, Puni and Ponte Brolla. For the sake of clarity, Buseno and Cauco are not shown as the results are very close to the efficiency plot obtained for Puni. There is a big difference between the red and blue Pareto frontiers, which are, respectively, obtained by considering all IHAs, and only those producing the highest variability (Table 4). This is particularly evident for Puni and Ponte Brolla power plants.

For Puni and Ponte Brolla, the red Pareto Frontier is almost horizontal, suggesting that assigning equal weight to the hydrological indicators will result in a Pareto Frontier that is independent of the water allocation scenario. Therefore, the 

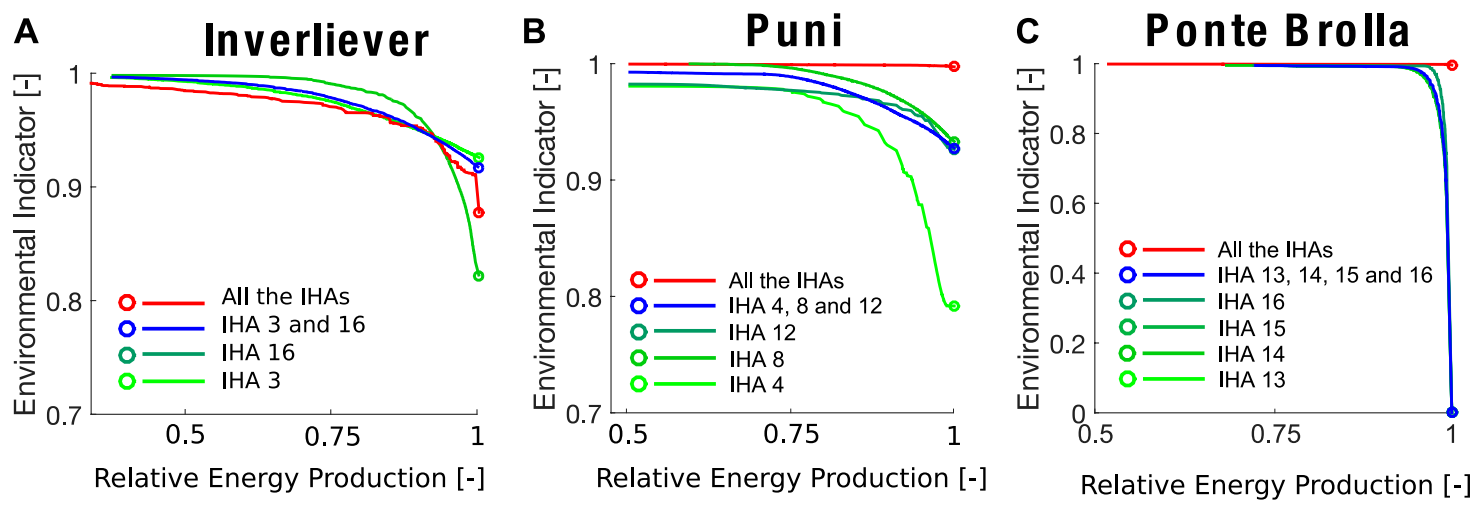

FIGURE 5 | Contribution of the IHA with the highest variability identified in Table $\mathbf{4}$ to compute the new Pareto Frontier (blue curve). The red curve represents the original Pareto Frontier that would be obtained if all the IHAs were taken into account. The dots represent $Q_{m f r}$ policies.

TABLE 4 | Indicators of Hydrologic Alteration showing the highest variability.

\begin{tabular}{lll} 
Station & IHA Number & \multicolumn{1}{c}{ Definition } \\
\hline Buseno & IHA \#3 & Mean flow rate value for March \\
& IHA \#16 & Annual maximum 3-days mean \\
Cauco & IHA \#17 & Annual minimum 7-days mean \\
& IHA \#3 & Mean flow rate value for March \\
& IHA \#16 & Annual maximum 3-days mean \\
Inverliever & IHA \#17 & Annual minimum 7-days mean \\
Puni & IHA \#3 & Mean flow rate value for March \\
& IHA \#16 & Annual maximum 3-days mean \\
& IHA \#4 & Mean flow rate value for April \\
Ponte Brolla & IHA \#8 & Mean flow rate value for August \\
& IHA \#12 & Mean flow rate value for December \\
& IHA \#13 & Annual minimum 1-d mean \\
& IHA \#15 & Annual maximum 1-d mean \\
& IHA \#16 & Annual minimum 3-days mean \\
& & Annual maximum 3-days mean
\end{tabular}

weight of the majority of the indicators that are insensitive to flow release policies can blur the effect of the few sensitive ones. However, for Inverliever, the red and blue lines are very close.

Figure 6 shows the efficiency plots for the proportional policies for each of the 32 hydrological sub-indicators. Note that the IHAs determining the highest variability (i.e., the most influential ones for changing water allocation policy) are the same as those in Figure 5. This shows that not only the NPPs are affecting the IHAs but also that the results can be transposed to all the different classes of distribution policies. This leads to the recommendation of performing a preliminary sensitivity analysis in order to identify which sub-indicators should be selected for the sake of meaningfulness of the results.

\subsection{Sensitivity Analysis on the Fish Habitat Suitability Indicator}

Figure 7 shows the Pareto frontiers obtained when considering only the fish habitat suitability indicator to compute the environmental indicator. Each color corresponds to a different choice from Table 3 used to calculate the indicator. For all three cases, the alternative $\mathrm{Eco}_{V 3}$ provides the worst values for the environmental indicator. This is because a non-proportional allocation results in a vertical shift of the natural hydrograph, thus bringing flow conditions persistently very close to the WUA threshold. For Cauco (Figure 7B), there is a large difference between the green and red lines. Differences between the minimal flow release $Q_{m f r}$ and the threshold of the Weighted Usable Area are small for this particular case. As the non-proportional allocation ensures a minimal flow, the penalty calculated by the alternative $\mathrm{Eco}_{\mathrm{V} 2}$ is guaranteed to be small. For low flows, the hydrograph stays close to the threshold but never really goes lower. Buseno has a hydrograph similar to Cauco and results in a similar value for $Q_{m f r}$. The difference is that the value of the threshold is higher for Buseno, thus the difference between the threshold and the $Q_{m f r}$ is bigger.

\subsection{Influence of Hydrograph Ergodicity and Sequence Length on the Pareto Frontier}

In the IHA method, Richter recommends using a set of at least $20 \mathrm{y}$ of hydrological data to evaluate annual variations of the flow regime. This can be a problem if the river is not monitored or if no gauging station is installed in a nearby catchment. For the Inverliever SHP, only $6 \mathrm{y}$ of data were available so a synthetic 20-y hydrograph was reconstructed using the technique described in Sensitivity analysis, which gives a statistically equivalent flow discharge time series.

For purely ergodic and stationary time series of infinite length, computing the Pareto Frontier starting from different discharge time sequences would reasonably not produce any appreciable change in the resulting Frontier. However, the series have a limited length and the effect of this on the Frontier is not obvious. Hence, 20 alternative hydrograph scenarios were generated and used to estimate the dependency of the Pareto Frontier on the length of the series. The newly generated synthetic hydrographs were found to have almost the same statistical properties as the original, and thus the limited length of the series has a minimal influence on the resulting efficiency plot. 


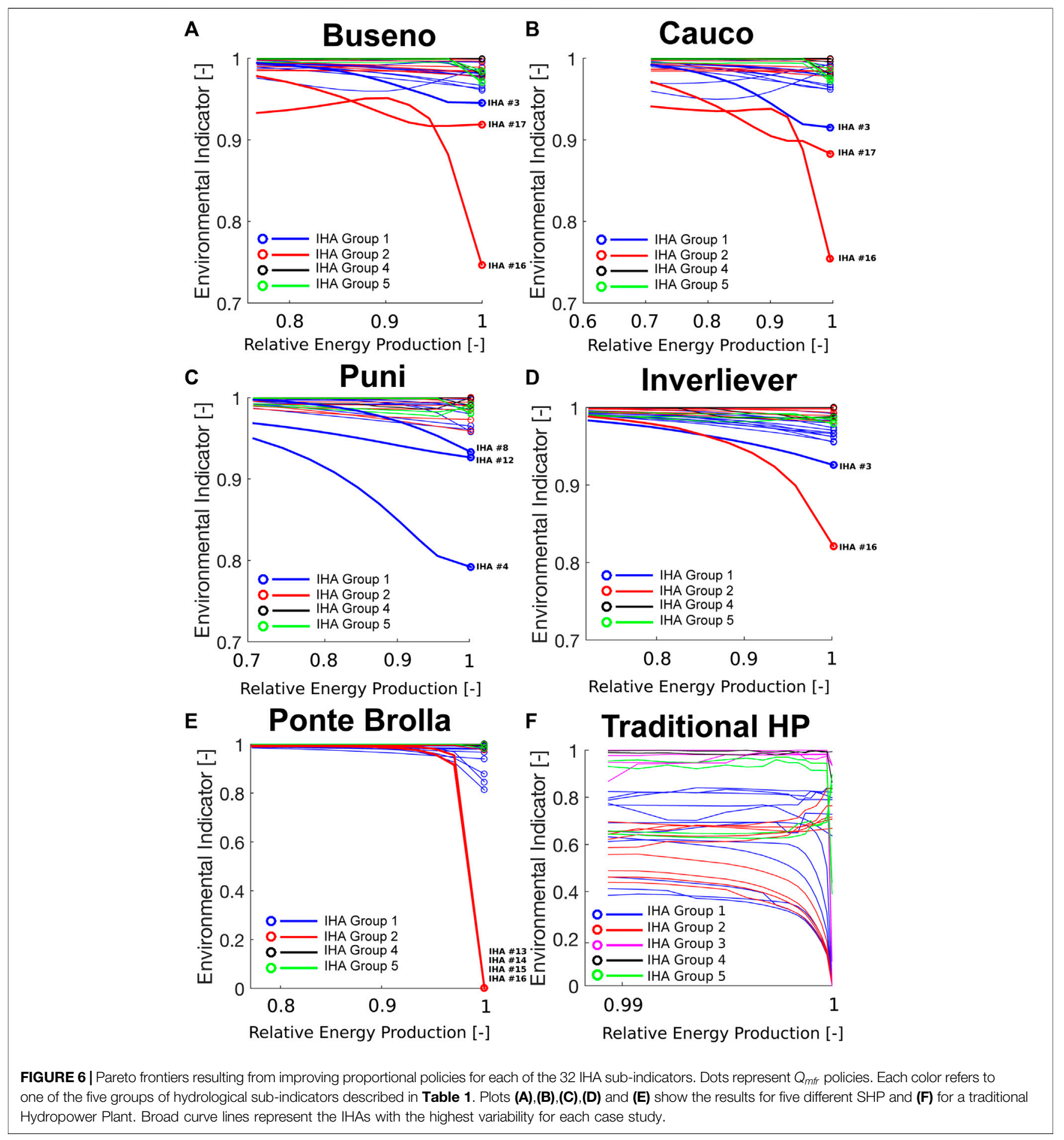

Figure 8 shows the Pareto frontiers resulting from using 20 statistically equivalent discharge sequences obtained from the PAR nonlinearly filtered model. The red line corresponds to the mean Pareto Frontier and the red circle is the average proportional policy with a percentage of $30 \%$. Provided that statistically equivalent hydrograph signals are used, the effect of limited length enhances hydrograph uncertainty. The resulting
Pareto frontiers appear therefore distributed around the mean within a band of about 3 percentile points.

\section{DISCUSSION}

In this work we chose to build the efficiency plot by considering the energy production and not the benefit as a key quantity. The 

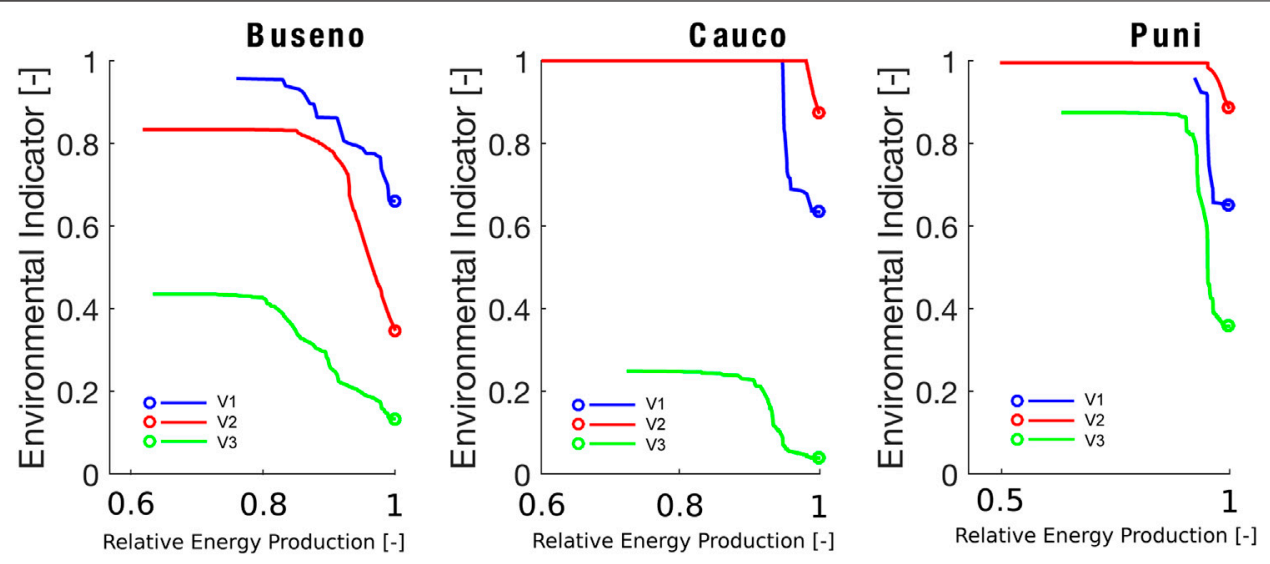

FIGURE 7|Pareto Frontier plots for non-proportional policies for the different versions of the fish indicator Eco $V_{1}$ (blue), Ecov2 (red) and Eco $v_{3}$ (green) for the three SHPs of Buseno, Cauco and Puni. Dots represent $Q_{m f r}$ policies.

advantage of doing this is that this choice will remove any dependency on economic market fluctuations and is more aligned with the unbiased estimate of the actual improvement that could be achieved in view of future energy transition strategies (e.g., the Swiss Energy Strategy 2050). More than for SHP, this can be an issue for traditional hydropower in that storage capacity clearly influences operational decisions. Notwithstanding this, this work provides a quantitative analysis of the global performance of hydropower, which might help identifying the best improvement strategy in view of the future transition towards sustainable energy sources (Willmott et al., 2016). This is also key with respect to the renewal of concession to SHP operating sustainable and globally efficient production policies.

\subsection{Importance of the Weighting in Building the Hydrological Indicator}

The sensitivity analysis highlights the impact of weighting the IHA indicators to compute the aggregated hydrological indicator.

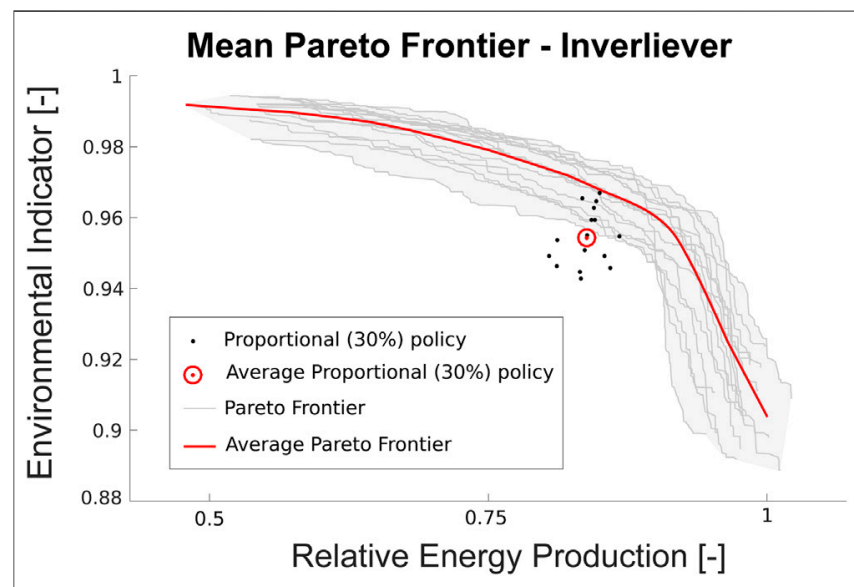

FIGURE 8 | Mean Pareto Frontier (red curve) and 30\% proportional policy (red dots) resulting from the average of 20 different generations of the 20-y hydrograph for the Inverliever SHP.
This is more important for computing the performance of SHPs than for traditional hydropower where more or less all indicators have a strong impact on the efficiency plot and can therefore all be weighted equally. Indeed, for the five SHP case studies, most of the IHA indicators (especially from Group 4 and 5) show little or no variation as a function of the water allocation policy. After aggregation, we obtained a horizontal Pareto Frontier very close to the natural regime, which would lead to a misinterpretation of the real performance of the adopted redistribution policy. Group 1 and 2 indicators are more affected by the change in water regime in the case of SHP, and IHA number 16 is common to 4 cases out of 5. A pre-sensitivity analysis would therefore help identify which IHAs are more suitable to represent the impact that the exploitation of a specific stream has on the components of the hydrologic regime.

A limit of the streamflow model used to compute the Pareto Frontier is that each case has to be considered separately and the weighting of the indicators is arbitrary. On the one hand, if one considers only the indicators that are strongly affected by the redistribution rules and removes the others, then the resulting Pareto Frontier will be pessimistically assessed because part of the information of the hydrologic regime variability will not be considered. On the other hand, if equal weight is given to each indicator, then the Pareto Frontier is hardly affected by the redistribution policies, and will result in an optimistic performance with a value very close to unity.

\subsection{Impact of the Fish Habitat Suitability Indicator on the Pareto Frontier}

The Fish Habitat Suitability indicator is generally considered important to complement the information from the hydrological indicators and build a comprehensive environmental indicator. The computation of the WUA curve requires river surveys, hydraulic measurements and significant computational power. Fish Habitat Suitability is however biased if used alone because of its dependence on the WUA threshold. This makes it representative of particular ecological species and it should therefore receive a lower weight in most cases unless diversely 
suggested by other considerations. Moreover, after aggregation, the Fish Habitat Suitability appears to have a stronger influence on the Pareto Frontier than the aggregated hydrological indicator. Each WUA curve is calculated for a specific species and a specific life stage. This would unfairly assess the importance of fish species with respect to other aquatic and terrestrial species that populate the riparian zone and whose adaptation to changing flow regime is better represented by indicators assessing the variability of the hydrological regime.

The differences between the three alternative indicators $\left(E c o_{V 1}, E c O_{V 2}, E c o_{V 3}\right)$ in terms of the values produced and their ranges (Figure 7) underscore the difficulty in creating a consistent indicator based only on the WUA curve. This is the case since, most of the time, the location of the break-point on the curve is not obvious and its position might possibly be biased towards high flows by the discretization scheme of numerical hydrodynamic models. An improvement of the WUA curve was recently proposed by Niayifar et al. (2018). They suggest that taking in-stream obstacles such as macroroughness elements into account results in a more accurate description of the WUA curve with an enhanced break-point at lower flow rates. This suggestion is valuable as it results in increased production without environmental consequences. However, issues related to sediment discontinuity (Gabbud and Lane, 2016) and hydro- and thermo-peaking were not considered and should be incorporated in future studies.

\subsection{Uncertainty of the Discharge Time Sequences}

Another major issue in assessing hydropower performance is hydrological data availability. While data collection and monitoring are important preliminary tasks for traditional hydropower, the design of SHPs often tolerates qualitatively approximated data. This is because the absence of storage in such plants precludes the need for accurate hydrological and economic forecasts to steer the production policy. However, incomplete data availability hampers evaluation of the longterm global performance of SHPs. Above, we proposed a simple time-series analysis-based model to generate statistically equivalent hydrological time sequences. The comparison of the IHA indicators between the modeled and the measured time series was shown to be a straightforward way to consider nonlinear dependencies in the data structure that would not be taken into account by linear autoregressive models. This modeling approach allowed for computation of the variability of the Pareto Frontier due to the use of short and non-perfectly ergodic time sequences. However, depending on information availability, other streamflow modeling approaches are possible. In particular, where they are feasible we recommend use of physically-based (either spatially distributed or semi-distributed) hydrological models such for example SWAT (https://swat.tamu.edu), or similar.

\section{CONCLUSION}

This study shows that for SHPs, only few among all indicators of hydrological alteration are actually relevant to evaluate the performance of the adopted redistribution rules as far as its hydrological similarities with the natural flow regime are concerned. As a consequence, this simplifies (also computationally) the choice of the best redistribution rule. Moreover, two better alternatives to the currently used fish habitat suitability indicators are also presented. The two different alternatives to the fish habitat suitability indicators proposed by Razurel et al. (2016) improve and strengthen the model by applying a more accurate weighting of the penalty. This construction of the ecohydrological indicator is crucial for evaluation of the efficiency of water distribution rules. Indeed, the sensitivity analysis performed on six different case studies with different water regimes leads to the conclusion that the aggregation method has a strong impact on the assessed environmental performance of the flow release policies. Each indicator evaluates a specific aspect of the impact of the hydropower plant on the environment. Some of them are not relevant in every case as they depend strongly on the characteristics of the river or the type of system (run-of-the-river SHP or traditional dam). Thus, for SHPs, the monthly streamflow magnitude and the magnitude and duration of extreme flow events have the highest influence on the choice of the optimal distribution policies. Conversely, for the traditional hydropower plant with storage, all the IHA groups influence the efficiency plot, including the frequency of high and low pulses and the rate of water condition changes. It is recommended that a preliminary analysis identifying which indicators have to be selected is performed before running the model to find the optimal water allocation rules.

\section{DATA AVAILABILITY STATEMENT}

The datasets analyzed and generated for this study can be found in the Zenodo data repository (file:///https://zenodo.org/record/ 5059557\#.YN6beC0RrGY).

\section{AUTHOR CONTRIBUTIONS}

$\mathrm{PP}$ is the main originator and wrote the article. AN implemented the analyses for the dammed systems. PR implemented the analyses for small hydropower systems. RS implemented the hydrological model, generated the inflow data for the Inverliever power plant. $\mathrm{RF}, \mathrm{EW}$, and $\mathrm{DB}$ contributed to the structuring phase, read and edited the manuscript.

\section{ACKNOWLEDGMENTS}

The authors are grateful to Adrian Loening and Walter Gostner for providing data regarding the SHPs of Inverliever and Puni. 


\section{REFERENCES}

Abbasi, T., and Abbasi, S. A. (2011). Small Hydro and the Environmental Implications of its Extensive Utilization. Renew. Sustain. Energ. Rev. 15, 2134-2143. doi:10.1016/j.rser.2010.11.050

Abbasi, T., Premalatha, M., and Abbasi, S. A. (2011). The Return to Renewables: Will it Help in Global Warming Control?. Renew. Sustain. Energ. Rev. 15, 891-894. doi:10.1016/j.rser.2010.09.048

Almeida, R. M., Shi, Q., Gomes-Selman, J. M., Wu, X., Xue, Y., Angarita, H., et al. (2019). Reducing Greenhouse Gas Emissions of Amazon Hydropower with Strategic Dam Planning. Nat. Commun. 10, 4281. doi:10.1038/s41467-01912179-5

Arthington, A. H., Bunn, S. E., Poff, N. L., and Naiman, R. J. (2006). The challenge of Providing Environmental Flow Rules to Sustain River Ecosystems. Ecol. Appl. 16, 1311-1318. doi:10.1890/1051-0761(2006)016[1311:tcopef]2.0.co;2

Basso, S., and Botter, G. (2012). Streamflow Variability and Optimal Capacity of Run-Of-River Hydropower Plants. Water Resour. Res. 48. doi:10.1029/ 2012wr012017

Benejam, L., Saura-Mas, S., Bardina, M., Solà, C., Munné, A., and García-Berthou, E. (2016). Ecological Impacts of Small Hydropower Plants on Headwater Stream Fish: From Individual to Community Effects. Ecol. Freshw. Fish. 25, 295-306. doi:10.1111/eff.12210

Bloesch, J., Ortlepp, J., and Ortlepp, J. (2005). An Application of Physical Habitat Modelling to Quantify Ecological Flow for the Rheinau Hydropower Plant, River Rhine. rs 16, 305-328. doi:10.1127/lr/16/2005/305

Brockwell, P. J., Davis, R. A., and Calder, M. V. (2002). Introduction to Time Series and Forecasting, Vol. 2. Springer.

Capra, H., Breil, P., and Souchon, Y. (1995). A New Tool to Interpret Magnitude and Duration of Fish Habitat Variations. Regul. Rivers: Res. Mgmt. 10, 281-289. doi:10.1002/rrr.3450100221

Environment Agency (2013). Environmental Flow Indicator. Tech. Rep. January, Environment Agency. Available at: http://webarchive. nationalarchives.gov.uk/20140328104910/http://cdn. environmentagency. gov.uk/LIT_7935_811630.pdf"/.

Gabbud, C., and Lane, S. N. (2016). Ecosystem Impacts of Alpine Water Intakes for Hydropower: the challenge of Sediment Management. WIREs Water 3, 41-61. doi:10.1002/wat2.1124

Gorla, L., and Perona, P. (2013). On Quantifying Ecologically Sustainable Flow Releases in a Diverted River Reach. J. Hydrol. 489, 98-107. doi:10.1016/ j.jhydrol.2013.02.043

Hertwich, E. G., Gibon, T., Bouman, E. A., Arvesen, A., Suh, S., Heath, G. A., et al. (2015). Integrated Life-Cycle Assessment of Electricity-Supply Scenarios Confirms Global Environmental Benefit of Low-Carbon Technologies. Proc. Natl. Acad. Sci. USA 112, 6277-6282. doi:10.1073/pnas.1312753111

Lazzaro, G., Basso, S., Schirmer, M., and Botter, G. (2013). Water Management Strategies for Run-Of-River Power Plants: Profitability and Hydrologic Impact between the Intake and the Outflow. Water Resour. Res. 49, n-a. doi:10.1002/ 2013wr014210-

Lifshitz, E., and Landau, L. (1984). Statistical Physics (Course of Theoretical Physics, Vol. 5. Oxford: Elsevier

Maddock, I. (1999). The Importance of Physical Habitat Assessment for Evaluating River Health. Freshw. Biol. 41, 373-391. doi:10.1046/j.13652427.1999.00437.x

Manzano-Agugliaro, F., Taher, M., Zapata-Sierra, A., Juaidi, A., and Montoya, F. G. (2017). An Overview of Research and Energy Evolution for Small Hydropower in Europe. Renew. Sustain. Energ. Rev. 75, 476-489. doi:10.1016/j.rser.2016.11.013

Marler, R. T., and Arora, J. S. (2004). Survey of Multi-Objective Optimization Methods for Engineering. Struct. Multidisciplinary Optimization 26, 369-395. doi:10.1007/s00158-003-0368-6

Mathews, R., and Richter, B. D. (2007). Application of the Indicators of Hydrologic Alteration Software in Environmental Flow Setting1. JAWRA J. Am. Water Resour. Assoc. 43, 1400-1413. doi:10.1111/j.1752-1688.2007.00099.x

Meijer, K. S., Van der Krogt, W. N. M., and van Beek, E. (2012). A New Approach to Incorporating Environmental Flow Requirements in Water Allocation Modeling. Water Resour. Manage. 26, 1271-1286. doi:10.1007/s11269-011-9958-z

Milhous, R., Wegner, D., Waddle, T., Energy, W., Team, L., Flow, I., et al. (1984). User's Guide to the Physical Habitat Simulation System (PHABSIM).
Department of the Interior, US Fish and Wildlife Service. Available at: http://pubs.er.usgs.gov/publication/fwsobs81_43"/.

Moyle, P. B., and Mount, J. F. (2007). Homogenous Rivers, Homogenous Faunas. Proc. Natl. Acad. Sci. 104, 5711-5712. doi:10.1073/pnas.0701457104

Niayifar, A., Oldroyd, H. J., Lane, S. N., and Perona, P. (2018). Modeling Macroroughness Contribution to Fish Habitat Suitability Curves. Water Resour. Res. 54, 9306-9320. doi:10.1029/2018wr022860

Niayifar, A., and Perona, P. (2017). Dynamic Water Allocation Policies Improve the Global Efficiency of Storage Systems. Adv. Water Resour. 104, 55-64. doi:10.1016/j.advwatres.2017.03.004

Payne, T. R. (2003). "The Concept of Weigthed Usable Area as Relative Suitability index," in Proceedings of International IFIM Users Workshop (CD), 1-5. Available at: https:// www.noaa.gov/sites/default/files/legacy/document/2020/Oct/07354626138.pdf/.

Perona, P., Dürrenmatt, D. J., and Characklis, G. W. (2013). Obtaining Natural-like Flow Releases in Diverted River Reaches from Simple Riparian Benefit Economic Models. J. Environ. Manag. 118, 161-169. doi:10.1016/j.jenvman.2013.01.010

Petts, G. E. (2009). Instream Flow Science for Sustainable River Management. JAWRA J. Am. Water Resour. Assoc. 45, 1071-1086. doi:10.1111/j.17521688.2009.00360.x

Poff, N. L., Olden, J. D., Merritt, D. M., and Pepin, D. M. (2007). Homogenization of Regional River Dynamics by Dams and Global Biodiversity Implications. Proc. Natl. Acad. Sci. 104, 5732-5737. doi:10.1073/pnas.0609812104

Poff, N. L., Richter, B. D., Arthington, A. H., Bunn, S. E., Naiman, R. J., Kendy, E., et al. (2010). The Ecological Limits of Hydrologic Alteration (ELOHA): a New Framework for Developing Regional Environmental Flow Standards. Freshw. Biol. 55, 147-170. doi:10.1111/j.1365-2427.2009.02204.x

Razurel, P., Gorla, L., Crouzy, B., and Perona, P. (2016). Non-proportional Repartition Rules Optimize Environmental Flows and Energy Production. Water Resour. Manage. 30, 207-223. doi:10.1007/s11269-015-1156-y

Razurel, P., Gorla, L., Tron, S., Niayifar, A., Crouzy, B., and Perona, P. (2018). Improving the Ecohydrological and Economic Efficiency of Small Hydropower Plants with Water Diversion. Adv. Water Resour. 113, 249-259. doi:10.1016/ j.advwatres.2018.01.029

Richter, B. D., Baumgartner, J. V., Powell, J., and Braun, D. P. (1996). A Method for Assessing Hydrologic Alteration within Ecosystems. Conservation Biol. 10, 1163-1174. doi:10.1046/j.1523-1739.1996.10041163.x

Robson, a., Cowx, I. G., and Harvey, J. P. (2011). Impact of Run-Of-River HydroSchemes upon Fish Populations. Edinburgh, Scotland: Environment, 1-71. Available at: http://www.sniffer.org.uk/knowledge-hubs/resilient-catchments/ water-frameworkdirective-and-uktag-co-ordination/impact-run-river-hydroschemes-upon-fish-populations/.

Sepa (2014). Guidance for Developers of Run-Of-River Hydropower Schemes. Tech. rep., Scottish Environment Protection Agency. Available at: https://www.sepa. org.uk/media/383805/guidance-_for_developers_of_run_of_river_hydropower_ schemes.pdf/.

Willmott, E., Gosling, R., and Pattullo, S. (2016). "10 Years of Licensing Run of River Hydropower Schemes in scotland - an Appraisal," in HydroES

Conflict of Interest: Authors PP, RF and EW were employed by the company HOLINGER AG.

The remaining authors declares that the research was conducted in the absence of any commercial or financial relationships that could be construed as a potential conflict of interest.

Publisher's Note: All claims expressed in this article are solely those of the authors and do not necessarily represent those of their affiliated organizations, or those of the publisher, the editors and the reviewers. Any product that may be evaluated in this article, or claim that may be made by its manufacturer, is not guaranteed or endorsed by the publisher.

Copyright (๑) 2021 Perona, Niayifar, Schwemmle, Razurel, Flury, Winz and Barry. This is an open-access article distributed under the terms of the Creative Commons Attribution License (CC BY). The use, distribution or reproduction in other forums is permitted, provided the original author(s) and the copyright owner(s) are credited and that the original publication in this journal is cited, in accordance with accepted academic practice. No use, distribution or reproduction is permitted which does not comply with these terms. 